\title{
Conceptual Design of an Environment for Systems Engineering Education
}

\author{
Jack Ring \\ Dennis Buede \\ Fred Bolling \\ Innovation Management Inc. \\ Innovative Decisions, Inc. \\ University of Michigan-Dearborn \\ Copyright $\odot 2005$ Jack Ring, Dennis Buede and Fred Bolling. Published and used by INCOSE with permission.
}

\begin{abstract}
A major problem in the teaching and learning of systems engineering is one of feedback concerning the quality of what we do: we often do not get any, and when we do, it is very late and imperfect. Accordingly, both initial learning and retention suffer. One of the few engineering domains involving complex systems and in which feedback is fast and quite accurate is racing, e.g., race cars!! Our proposed solution for the academic problem is a studio for modeling the "design, test, and operation of race cars". This laboratory stresses the breadth of systems and systems engineering rather than the depth that is typical of classes in other engineering disciplines. The proposed studio can be used for capstone design and project courses at both the undergrad and grad courses.
\end{abstract}

\section{Introduction}

Systems engineering (SE) is an engineering discipline that works with the customer and stakeholders to define needs, then specifies the engineering design problem for other disciplines of engineering. Concurrently, tests are specified and test results sufficient for updating the system model, both for initial acceptance and for operationally deployed versions of the system, are accumulated. System engineering is learned by the usual combination of lecture and laboratory. Unfortunately we are long on lecture and short on laboratory. In many schools the engineering students build a variety of small race cars. However the time limits and actual operational evaluation period is foreshortened by school schedules. Further, the participants tend to get lost in the details rather than discerning and experiencing the real systems aspects of the problem.

In contrast is the systems engineering learning that takes place in an automobile racing team such as a Formula 1 team in the FIA, a NASCAR team, a team at the Indianapolis 500 or even the upper classes of NHRA drag racing (recent record of more than $340 \mathrm{MPH}$ in one quarter of a mile). A race car is a complex yet still comprehensible system. A change 'here' affects more than one of the performance parameters and the effect of the change can be seen in a matter of seconds. Accordingly, experience on a race team is an excellent laboratory for a budding system engineer. Alternatively, a meaningful laboratory is observing and modeling the activities of a race team as a system.

Sponsored programs exist today involving student teams who design race vehicles and engage in short competition. However, these necessarily involve lots of development, test and administrative activities, thusly demonstrating the downstream $90 \%$ of a systems project rather than the front end $10 \%$.

The focus of a racing team is to safely win the race, which is much more well defined than almost any other system. So grand systems engineering phases like "operationally deployed 
versions of the system" simply mean ... the driver and the car on the track. The supersystem involves the sponsor, the organization that arranges hosted races over a system, the race track and whatever weather shows up on the day of the race, television and other coverages, endorsements and many other interrelated parcels. A major subsystem brings forth the pit crew, spotters, an experienced team boss and all other support people behind the car while it is immediately on the track. Further back in time is all the experienced-based technology challenged to push the driver, the car, and the team into a champagne-drenched winner's circle.

We are aware of all these and see them as one of the most exciting systems for studying and understanding just what a system is. When you do not win today, you revisit all the details to tweak out extras, which requires that you really know what the system is. But can you imagine a team, out of the money, wanting to have students with clipboards and distant professors underfoot while they fine tune for the next race.

Even so we thought how great it would be to have a coordinated trade-off structure to help the racer. At the same time think of the teaching potential for the 18 to 24 year old BS and MS students in systems engineering. At the least, picture some systems-related videos scripted and shot for all the involvements in winning the race. How do drivers interact everywhere - or do they? How far will sponsors go? What changes are too massive to get accomplished before the next race? When these and hundreds of other questions are asked, you learn. But producing these videos would require almost the same effort as setting up a studio. A second reality block.

So we thought it would be interesting to ignore all difficulties and assume the studio had been created. Then, to ask what do we learn, and how. Further, to wonder if this big answer is not possible (i.e., the studio) what are the small steps which would allow us to eat the whole elephant one bite a time.

The balance of this paper addresses Learning, Summary of Our Concept, ConOps of a Design Studio and Conclusion. References are provided as well. In Learning, in order to be clear about what we are trying to accomplish with variegated students, we examine Learning from Bloom's Taxonomy of outcomes, then from Isachsen's and Kolb's categories of student leaning styles thus implications on the learning environment, especially on the value of feedback, and finally from Ring's systems view of the 25 factors in an ideal learning environment In the Summary of Our Concept we make the case for how racing provides meaningful problem space and fast feedback as well as the very valuable aspect of excitement and enthusiasm. In the ConOps we summarize a few of the many ways a student would experience that Studio. In Conclusions we assert the value that a Design Studio add to the SE curriculum and learning experience.

\section{Learning}

Bloom, who headed a group of educational psychologists in 1956, is credited with a classification of levels of intellectual behavior important in learning. (http://www.officeport.com/edu/blooms.htm). Bloom identified six levels within the cognitive domain, from the simple recall or recognition of facts, as the lowest level, through increasingly more complex and abstract mental levels, to the highest order which is classified as evaluation. Verb examples that represent intellectual activity on each level are listed here. 
1. Knowledge: arrange, define, duplicate, label, list, memorize, name, order, recognize, relate, recall, repeat, and reproduce state.

2. Comprehension: classify, describe, discuss, explain, express, identify, indicate, locate, recognize, report, restate, review, select, and translate,

3. Application: apply, choose, demonstrate, dramatize, employ, illustrate, interpret, operate, practice, schedule, sketch, solve, use, and write.

4. Analysis: analyze, appraise, calculate, categorize, compare, contrast, criticize, differentiate, discriminate, distinguish, examine, experiment, question, and test.

5. Synthesis: arrange, assemble, collect, compose, construct, create, design, develop, formulate, manage, organize, plan, prepare, propose, set up, and write.

6. Evaluation: appraise, argue, assess, attach, choose compare, defend estimate, and judge.

Bloom found that over $95 \%$ of the test questions students encounter require them to think only at the lowest possible level...the recall of information. As teachers we tend to ask questions in the "knowledge" category $80 \%$ to $90 \%$ of the time. Engineering programs emphasize homework and test questions in the Application and Analysis categories. Real world engineering activity requires Synthesis and Evaluation. The six question categories defined by Bloom et al. (1956) are shown in Figure 1.

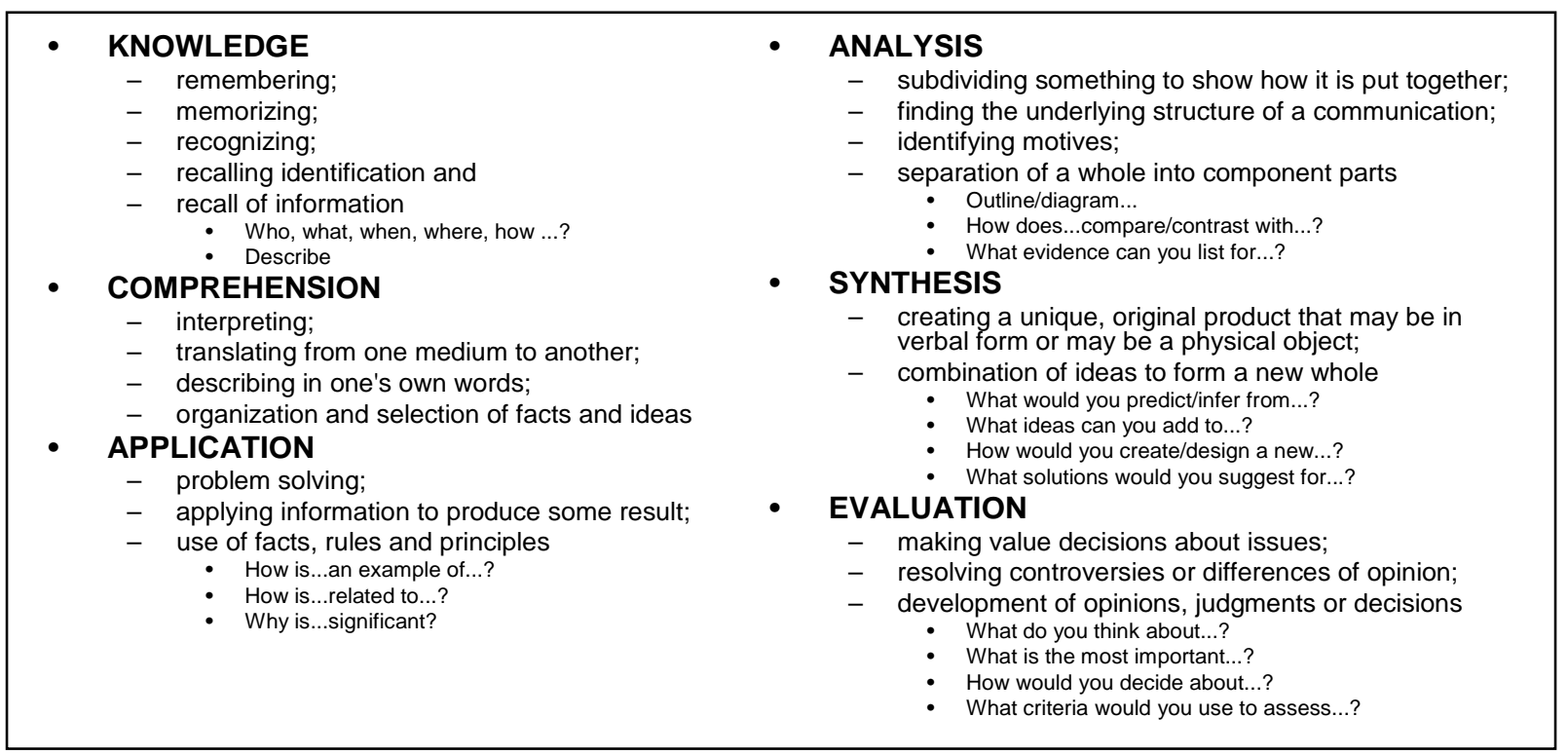

Figure 1. Question Associated with Six Levels of Learning

The proposed D\&T Studio would provide an excellent environment for asking Analysis, Synthesis and Evaluation kinds of questions about real world experiences.

\section{Summary of the SE Studio Concept}

A sample of the key SE principles that B.S. and M.S. graduates should have learned are:

1. The value of having a common language that spans stakeholders, engineers and specialists 
2. Defining the problem system before moving to the solution system. (or The criticality of modeling outside the system before progressing inside.

3. Applying systems engineering to the test system as well as to the solution system.

4. The need for a few key determinants of system success to guide all trade-offs and judge all design proposals and design change proposals.

5. Several reliable methods for determining whether the solution is susceptible to unintended consequences.

6. Seeing behavior of a system vs. form.

7. Orchestrating multiple, overlapping changes.

An important aspect of this concept is that the student learns more, faster about SE when they approach an actual system in the reverse of its life cycle. That is, observing and evaluating a system in operation then in test, then in assembly, then in design of components, then in change control deliberations, then in system architecture and design sessions, and finally in analysis of mission challenges (e.g., characteristics of the race track and competitors).

We propose to form a system that will leverage automobile racing to accelerate Learning about SE. The content and structure of the new system includes the race vehicle, situations and teams of people. The Learning Scenario includes: lecture, lab/project, capstone, practicum, apprenticeship, etc. The design studio equipment includes realistic race cars and track, access to race team personnel, simulation programs, and constraints on all resources. The SE focus in this design and testing studio would be on modeling, not for the purpose improving the engine or the suspension but for the purpose of addressing the trade offs across all specific areas in order to win the race. There are many known trades that the systems or chief engineer address.

What do we need??!!! Resources include a collection of IT with a wide range of models and simulation tools, a race/test/training track with race cars near a university with an SE program that is excited about such an opportunity, and a core staff with racing and SE experience,. Above this, interaction with a real race team is very desirable.

This design and testing studio would be hosted by a university but based at a race track (or test/training track); would be primarily focused on modeling and simulation tools for solving the SE problem at the system, supersystem and subsystem levels, but would have race cars available for experiential reinforcement; would be used by visiting students from any university; and would be staffed by rotating faculty members from many universities. (Note, such a facility would be a great resource for conducting research on design processes, methods, and techniques.) As mentioned earlier, racing provides a domain with nearly immediate and highly accurate feedback. While very few engineering students have experience with race cars, they all have experience at some level with cars. In addition, race cars provide a reasonably complex and precision system with many possible boundaries and high interaction with its environment. In addition, there is great design sophistication within the other engineering disciplines that participate in solving this engineering problem; yet the SE aspect of race car design is often still considered an "art”.

With these resources, what can we do? Accelerate and extend the education of SE students about the critical concepts that distinguish excellent systems engineers from average systems 
engineers. There will be spin offs to other engineering disciplines and to improving the quality of engineers in current practice. Finally, such a design and testing studio can provide valuable publicity for SE as a profession.

\section{SE Studio ConOps}

The SE scenario can be seen in the ISO Standard 15288 for System Life Cycle Processes and EIA Standard 632. Development of systems engineering practitioners seems to happen best from right to left on the system life cycle chart. That is, progressive engagements in:

A. Observing and evaluating the System in its Operations situations and concluding

a. What happened and when, in any one and all of several subsystems such as powertrain, traction and suspension, aerodynamics, driver environment, pit stop sequences and timings, etc.

b. What kind of pattern was exhibited? (cause, effect, association, coincidence)

c. Were the results good or bad or insignificant in each pattern?

d. Use Root Cause Analysis and appropriate Intent to suggest a capability change

B. Observing and evaluating the System in its Test situations and concluding

a. What happened and when, in any one and all of several subsystems such as powertrain, traction and suspension, aerodynamics, driver environment, pit stop sequences and timings, etc.

b. What kind of pattern was exhibited? (cause, effect, association, coincidence, e.g. what does the dynamometer profile predict about acceleration with different gear ratios and tire diameters? What does the dynamometer profile say about intake air flow? How can input air flow be measured under track conditions when the vehicle is drafting?)

c. Were the results good or bad or insignificant in each pattern?

d. Use Root Cause Analysis and appropriate Intent to suggest a capability change

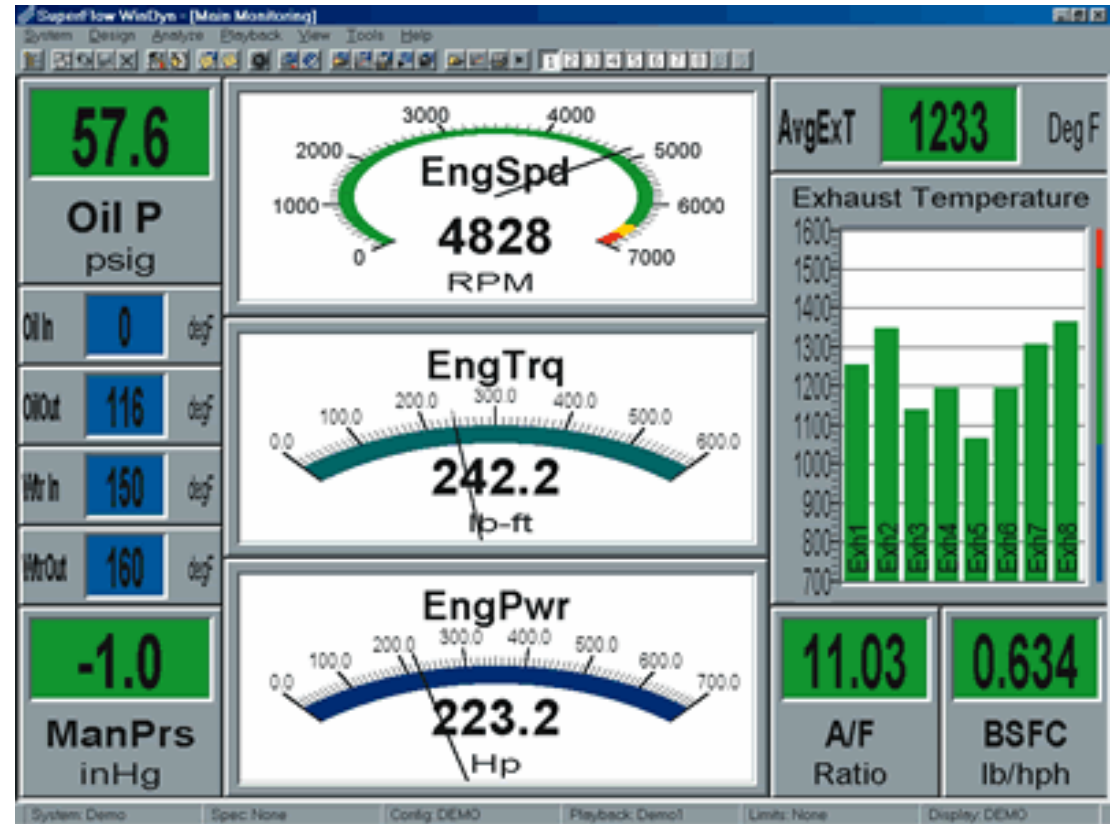

Figure 3. Engine Dynamometer Control Console

A dynamometer test readout contains data concerning 15 to 20 aspects of the engine that can be important. Insightful comparison of two tests with changes in controlled variables and/or configuration is the basis for a five to ten page analysis report by a student. 
C. Observing system in its integration test phase, and change management decisions

D. Participating in system component design change and change effect verification

E. Participating in a system capability change idea and identifying which components must undergo design change

F. Participating in system component development or acquisition and qualification

G. Participating in System Design

H. Participating in system concept formulation (including the definition of the problem)

We note that the typical SE curriculum sequence does not align well with this learning sequence.

As an example, the dynamometer is used to observe engine torque at various engine RPM's and at various accelerations (delta RPM per second increase) thus horsepower. Torque is determined not only by engine configuration (camshaft profile and phase, carburetor-manifold combinations, exhaust sizes and lengths) but also by controlled variables such as air-fuel ratio and ignition timing. A typical test operations console is shown in Figure 3.

In the professional racing business each lap is an experiment. The key to winning is observing more objectively and comprehending more effectively than the competition plus a little luck. Many hours are spent 'dialing in" the car to achieve the best possible match between the car, the driver, and the track. (Note, we cannot forget the importance of the driver in this process; great drivers will year after year with a wide range of cars.) This "dialing in" process means harmonizing the one hundred or so factors that effect one another on the track. A representative scenario might be:

1. Knowledge Base Updated

1.1. from last race

1.2. from component engineering

1.3. from people evaluations

2. Race Preparations

2.1. Issues and Intents (for learning and resolutions)

2.1.1. Car configuration

2.1.2. Team configuration

2.1.3. Inspection

2.1.4. Final preps and checks and double checks

2.2. Test Runs

2.2.1. Run 1 (focus on topic A)

2.2.1.1.Analysis

2.2.1.2.Adjustments

2.2.2. Run 2 (focus on topic B)

2.2.2.1.Analysis

2.2.2.2.Adjustments
2.3. Qualification runs

2.4. Learning 'personality' of car on this track

2.4.1. Ready

2.4.2. Driver's Meeting

2.4.3. Pit configuration

3. Race

2.4.4. Car Configuration

3.1. Race Management Plan

3.2. Green Flag

3.3. Driver Behavior

3.4. Driver reports

3.5. Instrumentation Reports

3.6. Context observations

3.7. Pit Stops

3.8. Checkered Flag

3.9. Inspection

4. After Action Review, Analysis and

Liar's Contest

A sample of what students would be able to do regarding a larger system such as Car, Situations and Teams (Crew) is: 
1. System Modeling

(External and Internal, Static and Dynamic)

2. System Simulation

Construct a Causal Loop simulation (aka Systems Dynamics) to clarify cardinality of relationships and the implications of time delays. Exercise scenarios to determine system stability limits.

Construct a Context-Sensitive simulation to understand the implicit interrelations and ideal gradient values for key interrelations. Also to understand how interrelations change with time, familiarity, successes, mistakes, etc.

3. Test Event planning, Instrumentation Analysis and Model Updating

(Operation, Change evaluation, Design ideas, SE cycle)

Examples of project activities that students would complete:

- Sample Design Issues Afforded by Racing Venue

- Describe the Concept of Operations of

- a race team

- a racing year

- a race week

- a green to checkered flag episode

- Place each of the following measures of effectiveness of a vehicle on a scale of 1 to 10 and cite three factors that influence the location you chose.

- Top Speed

- Acceleration profile

- Deceleration profile

- Reliability

- Ease of access for pit crew operations

- Square footage for sponsor signage.

- Place each of the following measures of effectiveness of a driver on a scale of 1 to 10 and cite three factors that influence the location you chose.

- Simultaneous minimization of lap time and wear

- Minimal catastrophic error in judgment

- Competency to observe vehicle and make recommendations or make on-track adjustments of engine, suspension, aerodynamic, etc.

Of course, the key activity for each team of students is to create a design effort for one of the many possible systems that can be considered: track monitoring system, pit crew system, car monitoring system, car and driver, etc.

\section{Conclusions}

The educational value of the proposed design studio fully fits into advanced theories about how people learn. The need to provide a simple objective for the system, to examine many possible slices as what the system or key subsystems are, and to obtain nearly immediate feedback about the effect of the engineering activities are critical education and our design studio concept. Racing has these elements in spades! 
The key questions that need to be addressed are:

1. How to create a win-win-win solution? From the car owner's perspective there is the interference vs. ROI (increase likelihood of winning, worth of civic duty, worth of own and sponsors' images)

2. How to organize universities so that many would allow their students and faculty to participate?

3. What Foundation might be interested in supporting this idea?

\section{References}

Bloom, B.S.; Taxonomy of Educational Objectives; Longman, New York; 1956, 1984.

Isachsen, O. and Berens, L. (1988) Working Together: A Personality-Centered Approach to Management, Neworld Press

Rechtin, E. and Maier, M. The Art of Systems Architecting, CRC Press, 1997

Ring, J. and Wymore, W. (2003). "Concept of Operations (ConOps) of a Systems Engineering Education Community (SEEC)”, Document No.: INCOSE-TP-2003-015-01 Version:0, 4 November 2003

\section{Bios}

Dennis Buede is VP and Chairman of the Board for a small decision and risk analysis firm (Innovative Decisions, Inc.) that is working primarily in the intelligence community. He has taught systems engineering at the undergraduate and graduate levels at George Mason University and Stevens Institute of Technology.

Jack Ring has significant experience with automotive systems and racing as long time associate with Bob Joehnck, Bonneville 200 MPH Club, and as VP for Corporate Development at the Edelbrock Corp. He was the first person to use aerospace telemetry on an SCCA race car, 1963, on the 614 Corvette built by Bob Joehnck and driven by Bob Bondurant. Also, he has held management and systems engineering positions with GE, Honeywell and IBM as well as mentoring a variety of high-tech startups and turn-arounds. Jack models enterprises as complex, adaptive, peopled systems.

Fred Bolling is professor of mechanical engineering at the University of Michigan-Dearborn where he teaches courses in Engineering Management and Automotive Systems Engineering. He held executive positions at Ford after many managerial jobs, following experience at Westinghouse and several universities, has been on the boards of several institutions and corporations and has participated in a wide range of national and international task forces. 